\title{
EXPOSIÇÃO AOS FATORES DE RISCO E VACINAÇÃO CONTRA HEPATITE B EM ESTUDANTES DO ENSINO MÉDIO DA REDE PÚBLICA
}

\section{Exposure to risk factors and hepatitis B vaccine among students attending public high schools

\author{
Exposición a los factores de riesgo y vacunación contra la hepatitis $B$ en estudiantes de
} secundaria de la red pública}

\author{
José Marcos de Jesus Santos \\ Universidade Federal de Sergipe - UFS - Lagarto (SE) - Brasil
}

Laíze Almeida dos Santos

Universidade Federal de Sergipe - UFS - Lagarto (SE) - Brasil

Flávia Márcia Oliveira

Universidade Federal de Sergipe - UFS - Lagarto (SE) - Brasil

\section{RESUMO}

Objetivo: Determinar a frequência de estudantes do ensino médio expostos à infecção viral e a situação vacinal contra hepatite B. Métodos: Estudo observacional descritivo transversal realizado entre os anos de 2014 e 2015. Para isso, foram aplicados 1.506 questionários, que continham questões referentes às características sociodemográficas e aos principais fatores de risco de exposição ao vírus, e analisados 240 cartões de vacina em sete colégios da rede estadual de quatro municípios do estado de Sergipe. Resultados: A maioria dos participantes era do sexo feminino $(987 ; 66 \%)$, com idade menor ou igual a 16 anos $(773 ; 51 \%)$ e ensino fundamental como o principal nível de escolaridade dos pais $(649 ; 43 \%$ pai; $758 ; 50 \%$ mãe). Os principais fatores de risco aos quais os estudantes estão expostos compreenderam o compartilhamento de materiais de manicure $(656 ; 44 \%)$ e de escova dental $(372 ; 24 \%)$, bem como relação sexual sem preservativo (281; 19\%). Apenas 513 alunos (34\%) não apresentaram nenhum fator de risco associado. Grande parte relatou possuir o cartão de vacina (1.054; 77\%), mas alguns desconhecem a própria situação vacinal $(430 ; 29 \%)$. Ao se considerar somente os cartões analisados (240), 212 registravam o esquema vacinal completo (88\%). Conclusão: Os estudantes do ensino médio regular da rede pública dos municípios avaliados configuram-se como vulneráveis à hepatite $\mathrm{B}$, pois foi observada elevada exposição aos fatores de risco e uma taxa de imunização abaixo da preconizada pelo Ministério da Saúde.

Descritores: Hepatite B; Estudantes; Fatores de risco; Vacinação.

\section{ABSTRACT}

Objective: To determine the frequency of high school students exposed to this viral infection and their hepatitis B immunization status. Methods: This is an observational and descriptive cross-sectional conducted between 2014 and 2015. A total of 1,506 questionnaires addressing sociodemographic characteristics and the main risk factors for exposure to the virus were administered. In addition, 240 vaccination records were analyzed in seven state-run schools of four municipalities in the state of Sergipe. Results: Participants were predominantly girls (987; $66 \%)$ and the majority was aged $\leq 16$ years $(773 ; 51 \%)$. Primary education predominated among the participants' parents (649; 43\% of fathers; 758; 50\% of mothers). The main risk factors to which the students were exposed included the sharing of manicure materials (656; $44 \%)$ and toothbrushes $(372 ; 24 \%)$ and sex without a condom (281; 19\%). Only 513 students (34\%) did not present any risk factors. Most of the students reported having a vaccination record (1,054; 77\%), but some were unware of their own immunization status (430; 29\%). Regarding the records analyzed (240), 212 students presented a full immunization status (88\%). Conclusion: The students of public high schools of the municipalities analyzed are vulnerable to hepatitis B given the high exposure to risk factors and the hepatitis $B$ immunization rate below the recommended by the Ministry of Health.

Descriptors: Hepatitis B; Students; Risk Factors; Vaccination. 


\section{RESUMEN}

Objetivo: Determinar la frecuencia de estudiantes de educación secundaria expuestos a infección viral y a la situación referente a la vacuna contra hepatitis B. Métodos: Se realizó un estudio observacional, descriptivo y transversal entre los años 2014 y 2015 . Para eso fueron aplicados 1.506 cuestionarios con preguntas sobre las características socio demográficas y los principales factores de riesgo para la exposición al virus y analizados 240 tarjetas de vacuna de siete colegios de la red estadual de cuatro municipios del estado de Sergipe. Resultados: La mayoría de los participantes era del sexo femenino ( $n=987 ; 66 \%)$ con edad igual o menos de 16 años ( $n=773$; $51 \%$ ) y educación básica como el principal nivel de escolaridad de los padres ( $n=649 ; 43 \%$ padre; $n=758 ; 50 \%$ madre). Los principales factores de riesgo para los cuales los estudiantes están expuestos fueron la acción de compartir los materiales de manicura ( $n=656 ; 44 \%)$ y de cepillo de dientes $(n=372 ; 24 \%)$ así como tener relación sexual sin condón $(n=281 ; 19 \%)$. Solamente 513 alumnos $(34 \%)$ no presentaron factor de riesgo asociado. Gran parte relató que tenía la tarjeta de vacuna $(n=1.054 ; 77 \%)$ pero algunos desconocen su propia situación referente a las vacunas ( $n=430 ; 29 \%)$. Considerando solamente las tarjetas analizadas (240), 212 registraban el esquema de vacuna completo (88\%). Conclusión: Los estudiantes de la educación secundaria regular de la red pública de los municipios evaluados se caracterizan como vulnerables a la hepatitis B pues ha sido observada elevada exposición para los factores de riesgo y una tasa de inmunización por debajo de la establecida por el Ministerio de la Salud.

Descriptores: Hepatitis B; Estudiantes; Factores de Riesgo; Vacunación.

\section{INTRODUÇÃO}

A hepatite provocada pelo vírus do sorotipo B (VHB) é uma das principais causas de doença hepática, principalmente nos países em desenvolvimento. Estima-se que cerca de 350 milhões de pessoas sejam portadoras crônicas do vírus no mundo ${ }^{(1)}$. No Brasil, entre 1999 a 2011, foram registrados 120.343 casos confirmados de hepatite B no Sistema de Informação de Agravos de Notificação (SINAN), sendo $54,2 \%$ entre homens ${ }^{(2)}$.

A forma mais comum de transmissão do VHB ocorre por meio das relações sexuais desprotegidas, cerca de $50 \%$ dos casos; seguida da utilização de objetos perfurocortantes não esterilizados e/ou reutilizados (drogas injetáveis, tatuagens, procedimentos de manicures, ambulatoriais, cirúrgicos, odontológicos, perfuração de orelha e colocação de piercings); e contaminação perinatal e intradomiciliar (compartilhamento de escova de dente e lâminas de barbear) ${ }^{(3)}$.

Considerando as formas de transmissão, a infecção pelo VHB pode acometer qualquer pessoa. Entretanto, alguns grupos populacionais estão particularmente mais expostos ao vírus em função dos hábitos comportamentais de risco - múltiplos parceiros sexuais, prática sexual insegura, uso de drogas injetáveis ${ }^{(4)}$, piercing e tatuagem ${ }^{(5)}$ - e das exposições ocupacionais, caso dos profissionais de saúde e policiais ${ }^{(6)}$. Além disso, o risco para infecção parece mais evidente em contextos sociais menos favorecidos $^{(7)}$ e na faixa etária entre 15 e 39 anos, cerca de $75 \%$ dos casos novos ${ }^{(8)}$.

No Brasil, as ações de imunização contra o Vírus da Hepatite B (VHB) iniciaram-se em 1989, nos municípios de Purus, Boca do Abre e Lábrea, na Região Amazônica, em crianças com até dez anos e profissionais de saúde ${ }^{(6)}$. Em 1998/1999, ocorreu a implantação da vacina contra hepatite B para menores de um ano em todo o país. Ao longo dos anos, de forma gradativa, houve a ampliação da oferta da vacina para a maior parte da população, sendo que, no início de 2016, ampliou-se para todas as idades, independentemente das condições de vulnerabilidade social e comportamental ${ }^{(9)}$.

A partir da análise do contexto da hepatite B, os processos de promoção da saúde devem incluir atividades de educação por meio da ampliação do conhecimento da forma de transmissão da doença com foco nos cuidados com materiais potencialmente contaminados e uso de preservativos nas relações sexuais. Além disso, devem ser complementados com estratégias de prevenção da doença que envolve a identificação precoce e imunização das pessoas susceptíveis.

Portanto, o estudo objetivou determinar a frequência de estudantes do ensino médio expostos à infecção viral e a situação vacinal contra hepatite B.

\section{MÉTODOS}

Realizou-se um estudo observacional com abordagem descritiva do tipo transversal de setembro/2014 a junho/2015. A população-alvo foi constituída por estudantes matriculados no ensino médio regular da Diretoria Regional de Educação (DRE02) dos municípios de Lagarto, Riachão do Dantas, Simão Dias e Tobias Barreto, localizados em Sergipe, Brasil. Segundo informações da Secretaria de Estado da Educação de Sergipe, em 2014 constavam respectivamente 3.048, 514, 1.374 e 1.552 alunos matriculados nos colégios dos municípios acima citados.

Para avaliação da frequência à exposição aos fatores de risco de infecção pelo VHB, selecionaram-se 1.506 estudantes matriculados no ensino médio regular utilizando a amostragem por conveniência (erro amostral 5\%; nível de confiança 95\%; estimativa de 55\% dos estudantes expostos a um fator de risco de infecção, no mínimo). Posteriormente, analisou-se a situação vacinal por meio da visualização de 240 cartões de vacina dos indivíduos sorteados a partir dos 1.506 participantes da etapa 
anterior (erro amostral 5\%; nível de confiança 95\%; estimativa de $80 \%$ dos estudantes com esquema vacinal completo, no mínimo).

A abordagem metodológica envolveu um instrumento de coleta de dados composto por duas partes: questionário composto por questões objetivas relacionadas às características sociodemográficas (idade, sexo, moradia e escolaridade dos pais) e aos principais fatores de risco de exposição ao VHB descritos na literatura (prática sexual insegura, exposições percutâneas, compartilhamento de seringas ou escovas de dente, transfusão e/ou transplante) e formulário de extração de dados referentes ao cartão de vacinação (registro da imunização e número de doses da vacina contra hepatite B).

Para criação do banco de dados e codificação das variáveis do estudo utilizou-se o programa IBM SPSS Statistics 20®, a partir do qual se empregou a estatística descritiva para obtenção das frequências absoluta, relativa, média, desvio-padrão, mínimo e máximo.

A pesquisa ocorreu de acordo com as diretrizes e normas regulamentadoras preconizadas na Resolução ${ }^{\circ}$ 446/12 do Conselho Nacional de Saúde, sendo aprovada pelo Comitê de Ética em Pesquisa da Universidade Federal de Sergipe $\left(\mathrm{n}^{\circ}\right.$ do parecer 798.187).

\section{RESULTADOS}

A amostra de 1.506 estudantes matriculados no ensino médio regular da rede pública foi constituída por $66 \%$ dos participantes do sexo feminino $(\mathrm{n}=987)$ e com faixa etária $\leq 16$ anos $(\mathrm{n}=773)$, sendo a idade média igual a 16,1 $( \pm 0,3$ anos; mínimo=12 e máximo=27). Em relação à moradia, houve uma distribuição similar, sendo $50 \%$ localizada na zona rural $(\mathrm{n}=760)$ e $47 \%$ na urbana $(n=701)$. Constatou-se que, em relação ao perfil de escolaridade predominante dos pais, $43 \%$ dos pais $(n=649)$ e $50 \%$ das mães $(n=758)$ possuíam apenas ensino fundamental (Tabela I).

Tabela I - Variáveis sociodemográficas dos estudantes matriculados no ensino médio regular da rede pública estadual $(\mathrm{n}=$ 1.506) de Lagarto, Riachão do Dantas, Simão Dias e Tobias Barreto - Sergipe, Brasil, 2014-2015.

\begin{tabular}{|c|c|c|c|c|c|c|c|c|c|c|}
\hline \multicolumn{11}{|c|}{ Cidade/Município } \\
\hline & \multicolumn{2}{|c|}{ Lagarto } & \multicolumn{2}{|c|}{ Riachão do Dantas } & \multicolumn{2}{|c|}{ Simão Dias } & \multicolumn{2}{|c|}{ Tobias Barreto } & \multicolumn{2}{|c|}{ Total } \\
\hline & $\mathbf{n}$ & $\%$ & $\mathbf{n}$ & $\%$ & $\mathbf{n}$ & $\%$ & $\mathbf{n}$ & $\%$ & $\mathbf{n}$ & $\%$ \\
\hline \multicolumn{11}{|l|}{ Idade } \\
\hline$\leq 16$ anos & 468 & 52 & 85 & 46 & 82 & 69 & 138 & 45 & 773 & 51 \\
\hline$\geq 17$ anos & 422 & 47 & 96 & 52 & 35 & 30 & 163 & 54 & 716 & 48 \\
\hline N.R. & 12 & 1 & 2 & 2 & 1 & 1 & 2 & 1 & 17 & 1 \\
\hline \multicolumn{11}{|l|}{ Sexo } \\
\hline Feminino & 571 & 63 & 128 & 70 & 81 & 69 & 207 & 68 & 987 & 66 \\
\hline Masculino & 311 & 35 & 50 & 27 & 35 & 30 & 91 & 30 & 487 & 32 \\
\hline N.R. & 20 & 2 & 5 & 3 & 2 & 1 & 5 & 2 & 29 & 2 \\
\hline \multicolumn{11}{|l|}{ Moradia } \\
\hline Urbana & 326 & 61 & 60 & 33 & 52 & 44 & 263 & 87 & 701 & 47 \\
\hline Rural & 554 & 36 & 111 & 61 & 64 & 54 & 31 & 10 & 760 & 50 \\
\hline N.R. & 22 & 3 & 12 & 6 & 2 & 2 & 9 & 3 & 45 & 3 \\
\hline \multicolumn{11}{|c|}{ Escolaridade do pai } \\
\hline Analfabeto & 167 & 18 & 29 & 16 & 31 & 26 & 43 & 14 & 270 & 18 \\
\hline Fundamental & 380 & 42 & 71 & 39 & 56 & 48 & 142 & 47 & 649 & 43 \\
\hline Médio & 192 & 22 & 32 & 18 & 12 & 10 & 61 & 20 & 297 & 20 \\
\hline Superior & 48 & 5 & 19 & 10 & 2 & 2 & 26 & 9 & 95 & 6 \\
\hline N.S. & 107 & 12 & 26 & 14 & 17 & 14 & 29 & 9 & 179 & 12 \\
\hline N.R. & 8 & 1 & 6 & 3 & 0 & 0 & 2 & 1 & 16 & 1 \\
\hline \multicolumn{11}{|c|}{ Escolaridade da mãe } \\
\hline Analfabeta & 141 & 16 & 22 & 12 & 14 & 12 & 37 & 12 & 214 & 14 \\
\hline Fundamental & 450 & 50 & 83 & 45 & 74 & 63 & 151 & 50 & 758 & 50 \\
\hline Médio & 167 & 19 & 25 & 13 & 16 & 13 & 64 & 21 & 272 & 18 \\
\hline Superior & 46 & 5 & 28 & 15 & 5 & 4 & 24 & 8 & 103 & 7 \\
\hline N.S. & 85 & 9 & 19 & 10 & 9 & 8 & 25 & 8 & 138 & 9 \\
\hline N.R. & 13 & 1 & 6 & 3 & 0 & 0 & 2 & 1 & 21 & 2 \\
\hline
\end{tabular}

Nota: N.S. (Não sabe a resposta); N.R. (Não respondeu).

Fonte: Dados da pesquisa dos autores (questionários). 
Foram elencados fatores de risco relacionados à transmissão do VHB bem descritos na literatura, como tatuagem/piercing, compartilhamento de material de manicure e escova de dente, relação sexual sem preservativo, uso de drogas injetáveis e contato com sangue de outras pessoas no dia a dia e/ou por meio de transfusão ou transplante. A Tabela II mostra que as principais possíveis formas de exposição dos estudantes ao VHB estão relacionadas especialmente ao compartilhamento de materiais de manicure $(n=656 ; 44 \%)$ e escova de dente $(n=372 ; 24 \%)$, bem como ao contato com sangue de outras pessoas nos casos de ferimentos $(n=368 ; 24 \%)$. Além disso, uma parte considerável ( $n=281 ; 19 \%)$ já foi exposta ao VHB e outras doenças sexualmente transmissíveis (DSTs), visto que relataram relação sexual sem a utilização de preservativo. Por outro lado, a exposição relacionada aos procedimentos de tatuagem, piercing, transfusão de sanguetransplante e ao uso de drogas injetáveis foi bem reduzida $(\leq 3 \%)$.

Tabela II - Fatores de risco relacionados à exposição ao vírus da hepatite B em estudantes matriculados no ensino médio regular da rede pública estadual $(\mathrm{n}=1.506)$ de Lagarto, Riachão do Dantas, Simão Dias e Tobias Barreto - Sergipe, Brasil, $2014-2015$.

\begin{tabular}{|c|c|c|c|c|c|c|c|c|c|c|}
\hline & \multicolumn{8}{|c|}{ Cidade/Município } & & \\
\hline & \multicolumn{2}{|c|}{ Lagarto } & \multicolumn{2}{|c|}{$\begin{array}{c}\text { Riachão do } \\
\text { Dantas }\end{array}$} & \multicolumn{2}{|c|}{ Simão Dias } & \multicolumn{2}{|c|}{$\begin{array}{c}\text { Tobias } \\
\text { Barreto }\end{array}$} & \multicolumn{2}{|c|}{ Total } \\
\hline & $\mathbf{n}$ & $\%$ & $\mathbf{n}$ & $\%$ & $\mathbf{n}$ & $\%$ & $\mathbf{n}$ & $\%$ & $\mathbf{n}$ & $\%$ \\
\hline \multicolumn{11}{|l|}{ Tatuagem } \\
\hline Não & 863 & 96 & 176 & 96 & 116 & 98 & 291 & 96 & 1446 & 96 \\
\hline Sim & 29 & 3 & 4 & 2 & 2 & 2 & 11 & 3 & 46 & 3 \\
\hline N.R. & 10 & 1 & 3 & 2 & - & - & 1 & 1 & 14 & 1 \\
\hline N.S. & - & - & - & - & - & - & - & - & - & - \\
\hline \multicolumn{11}{|l|}{ Piercing } \\
\hline Não & 848 & 94 & 178 & 97 & 114 & 97 & 286 & 95 & 1426 & 95 \\
\hline Sim & 36 & 4 & 2 & 1 & 3 & 2 & 10 & 3 & 51 & 3 \\
\hline N.R. & 18 & 2 & 3 & 2 & 1 & 1 & 7 & 2 & 29 & 2 \\
\hline N.S. & - & - & - & - & - & - & - & - & - & - \\
\hline \multicolumn{11}{|l|}{ Transfusão/Transplante } \\
\hline Não & 782 & 87 & 163 & 89 & 109 & 92 & 262 & 87 & 1316 & 87 \\
\hline Sim & 3 & 1 & 2 & 1 & 1 & 1 & 4 & 1 & 10 & 1 \\
\hline N.R. & 59 & 6 & 4 & 3 & 3 & 4 & 16 & 5 & 81 & 5 \\
\hline N.S. & 58 & 6 & 14 & 7 & 5 & 3 & 21 & 7 & 99 & 7 \\
\hline \multicolumn{11}{|l|}{ Manicure } \\
\hline M. da manicure & 371 & 41 & 76 & 42 & 41 & 35 & 168 & 55 & 656 & 44 \\
\hline M. próprio / Não usa & 491 & 55 & 99 & 54 & 77 & 65 & 118 & 39 & 785 & 52 \\
\hline N.R. & 20 & 2 & 4 & 1 & - & - & 12 & 4 & 35 & 2 \\
\hline N.S. & 20 & 2 & 4 & 2 & - & - & 5 & 2 & 30 & 2 \\
\hline \multicolumn{11}{|c|}{ Compartilha escova dental } \\
\hline Não & 644 & 71 & 127 & 69 & 91 & 77 & 222 & 73 & 1084 & 72 \\
\hline Sim & 228 & 25 & 50 & 27 & 24 & 20 & 70 & 23 & 372 & 24 \\
\hline N.R. & 5 & 1 & 2 & 2 & 1 & 1 & 1 & 1 & 9 & 1 \\
\hline N.S. & 25 & 2 & 4 & 3 & 2 & 2 & 10 & 3 & 41 & 3 \\
\hline \multicolumn{11}{|c|}{ Relação sexual sem preservativo } \\
\hline Não & 688 & 76 & 148 & 81 & 97 & 82 & 239 & 79 & 1172 & 78 \\
\hline Sim & 182 & 20 & 29 & 16 & 16 & 14 & 55 & 18 & 281 & 19 \\
\hline N.R. & 25 & 3 & 2 & 1 & 2 & 2 & 4 & 1 & 33 & 2 \\
\hline N.S. & 7 & 1 & 4 & 2 & 3 & 2 & 5 & 2 & 20 & 1 \\
\hline \multicolumn{11}{|l|}{ Uso de drogas injetáveis } \\
\hline Não & 880 & 97 & 180 & 98 & 115 & 97 & 290 & 96 & 1465 & 97 \\
\hline Sim & 4 & 1 & 2 & 1 & 1 & 1 & 4 & 1 & 11 & 1 \\
\hline N.R. & 9 & 1 & - & - & - & - & 3 & 1 & 12 & 1 \\
\hline N. S. & 9 & 1 & 1 & 1 & 2 & 2 & 6 & 2 & 18 & 1 \\
\hline \multicolumn{11}{|c|}{ Contato com sangue de outras pessoas } \\
\hline Não & 587 & 65 & 110 & 60 & 81 & 69 & 166 & 55 & 944 & 63 \\
\hline Sim & 209 & 23 & 47 & 26 & 25 & 21 & 87 & 29 & 368 & 24 \\
\hline N.R. & 8 & 1 & - & - & 1 & 1 & 1 & 1 & 10 & 1 \\
\hline N.S. & 98 & 11 & 26 & 14 & 11 & 9 & 49 & 15 & 184 & 12 \\
\hline
\end{tabular}

Nota: N.S. (Não sabe a resposta); N.R. (Não respondeu). Fonte: Dados da pesquisa dos autores (questionários). 
Considerando o alto índice de respostas relacionadas ao risco de exposição ao VHB, realizou-se uma análise acumulativa, ou seja, foi verificada a frequência da presença de um ou mais fatores de risco para o VHB para cada indivíduo (Tabela III). Observou-se que apenas 513 estudantes (34\%) não apresentaram nenhum fator de risco associado, 469 apresentavam apenas 1 fator (31\%) e 524 apresentavam 2 ou mais fatores (43\%). Dessa forma, reforça a análise do comportamento de risco pela maioria dos adolescentes.

Tabela III - Fatores de risco acumulados referentes à exposição ao vírus da hepatite B nos estudantes matriculados no ensino médio regular da rede pública estadual $(\mathrm{n}=1.506)$ de Lagarto, Riachão do Dantas, Simão Dias e Tobias Barreto - Sergipe, Brasil, 2014-2015.

\begin{tabular}{|c|c|c|c|c|c|c|c|c|c|c|}
\hline \multirow{3}{*}{$\begin{array}{l}\text { Quantidade de fatores de risco aos quais o } \\
\text { indivíduo está exposto }\end{array}$} & \multicolumn{6}{|c|}{ Cidade/Município } & \multirow{2}{*}{\multicolumn{2}{|c|}{$\begin{array}{c}\text { Tobias } \\
\text { Barreto }\end{array}$}} & \multirow{2}{*}{\multicolumn{2}{|c|}{ Total }} \\
\hline & \multicolumn{2}{|c|}{ Lagarto } & \multicolumn{2}{|c|}{$\begin{array}{l}\text { Riachão do } \\
\text { Dantas }\end{array}$} & \multicolumn{2}{|c|}{ Simão Dias } & & & & \\
\hline & $\mathbf{n}$ & $\%$ & $\mathbf{n}$ & $\%$ & $\mathbf{n}$ & $\%$ & $\mathbf{n}$ & $\%$ & $\mathbf{n}$ & $\%$ \\
\hline 0 & 316 & 35 & 67 & 37 & 58 & 49 & 72 & 24 & 513 & 34 \\
\hline 1 & 275 & 30 & 55 & 30 & 29 & 25 & 110 & 36 & 469 & 31 \\
\hline 2 & 191 & 21 & 33 & 18 & 17 & 14 & 79 & 26 & 320 & 21 \\
\hline 3 & 85 & 9 & 23 & 12 & 8 & 7 & 32 & 10 & 148 & 10 \\
\hline 4 & 29 & 3 & 5 & 3 & 5 & 4 & 7 & 2 & 46 & 3 \\
\hline 5 & 5 & 1 & - & - & 1 & 1 & 2 & 1 & 8 & 1 \\
\hline 6 & 1 & 1 & - & - & - & - & - & - & 1 & - \\
\hline 7 & - & - & - & - & - & - & 1 & 1 & 1 & - \\
\hline 8 & - & - & - & - & - & - & - & - & - & - \\
\hline
\end{tabular}

Fonte: Dados da pesquisa dos autores (questionários).

Quanto à investigação da situação vacinal (Tabela IV), a maioria relatou que possuía o cartão de vacina ( $\mathrm{n}=1054 ; 77 \%)$ e apenas 873 dos estudantes (58\%) afirmaram que eram vacinados contra hepatite B. Ao se considerar, contudo, somente os cartões de vacina analisados durante a pesquisa $(n=240), 212$ registravam o esquema vacinal completo $(88 \%)$.

Tabela IV - Situação quanto ao cartão de vacina e vacinação contra o vírus da hepatite B, relatado $(\mathrm{n}=1.506)$ e/ou visualizado $(\mathrm{n}=240)$, dos estudantes matriculados no ensino médio regular da rede pública estadual de Lagarto, Riachão do Dantas, Simão Dias e Tobias Barreto - Sergipe, Brasil, 2014-2015.

\begin{tabular}{|c|c|c|c|c|c|c|c|c|c|c|}
\hline & \multicolumn{8}{|c|}{ Cidade/Município } & & \\
\hline & \multicolumn{2}{|c|}{ Lagarto } & \multicolumn{2}{|c|}{$\begin{array}{c}\text { Riachão do } \\
\text { Dantas }\end{array}$} & \multicolumn{2}{|c|}{ Simão Dias } & \multicolumn{2}{|c|}{$\begin{array}{l}\text { Tobias } \\
\text { Barreto }\end{array}$} & \multicolumn{2}{|c|}{ Total } \\
\hline & $\mathbf{n}$ & $\%$ & n & $\%$ & $\mathbf{n}$ & $\%$ & na & $\%$ & $\mathbf{n}$ & $\%$ \\
\hline \multicolumn{11}{|c|}{ Cartão de vacina (relatado) } \\
\hline $\operatorname{Sim}$ & 584 & 64 & 133 & 73 & 96 & 81 & 243 & 81 & 1054 & 77 \\
\hline Não & 100 & 12 & 28 & 15 & 5 & 5 & 32 & 10 & 165 & 12 \\
\hline N.S. & 218 & 24 & 22 & 12 & 17 & 14 & 28 & 9 & 285 & 11 \\
\hline \multicolumn{11}{|c|}{ Vacina contra VHB (relatado) } \\
\hline $\operatorname{Sim}$ & 633 & 43 & 94 & 51 & 55 & 46 & 91 & 30 & 873 & 58 \\
\hline Não & 108 & 12 & 20 & 11 & 11 & 9 & 64 & 21 & 203 & 13 \\
\hline N.S. & 161 & 45 & 69 & 38 & 52 & 44 & 148 & 49 & 430 & 29 \\
\hline \multicolumn{11}{|c|}{ Cartão de vacina (visualizado) } \\
\hline Sim & 152 & 17 & 26 & 14 & 4 & 4 & 58 & 19 & 240 & 16 \\
\hline Não & 750 & 83 & 157 & 86 & 114 & 96 & 245 & 81 & 1266 & 84 \\
\hline \multicolumn{11}{|c|}{ Vacina contra VHB (visualizado) } \\
\hline 3 & 147 & 97 & 25 & 96 & 3 & 75 & 37 & 64 & 212 & 88 \\
\hline 2 & 2 & 1 & - & - & - & - & 4 & 7 & 6 & 2 \\
\hline 1 & - & - & 1 & 4 & - & - & 7 & 12 & 8 & 3 \\
\hline- & 3 & 2 & - & - & 1 & 25 & 10 & 17 & 14 & 7 \\
\hline
\end{tabular}

Nota: N.S. (Não sabe a resposta).

Fonte: Dados da pesquisa dos autores (questionários). 


\section{DISCUSSÃO}

A maioria dos estudantes do presente estudo estava na fase da vida definida pela Organização Mundial da Saúde como adolescência ${ }^{(10)}$. Sabe-se que é uma etapa caracterizada por rápidas e diversas transformações, que elevam o nível de intensidade das emoções e vivências. Trata-se de um período em que se estabelece, de forma mais definitiva, a identidade, os padrões de comportamento e estilos de vida. A rebeldia e a indisciplina presentes nos adolescentes (10 a 19 anos) são explicadas pelas alterações que ocorrem nas redes neuronais do cérebro durante essa fase, ou seja, as conexões nervosas são reestruturadas com o intuito de preparar-se de forma definitiva para a vida adulta. Modificações associadas ao desenvolvimento dos sistemas subcorticais do cérebro, à autorregulação imatura e às alterações neuroendócrinas mediadas pelo eixo hipotalâmico-hipofisário relacionam-se com o aumento dos comportamentos de risco ${ }^{(11)}$. No entanto, torna-se fundamental ampliar o olhar para além das alterações biológicas através de uma análise mais profunda da relação do adolescente com o processo saúde-doença e seus determinantes sociais.

$\mathrm{Na}$ presente pesquisa, a identificação da existência de comportamentos de risco na maioria dos estudantes (66\%) relacionados à exposição ao VHB e uma frequência de imunização contra o vírus menor do que a preconizada pelo Ministério da Saúde (88\%) provocam uma reflexão ligada à teoria das representações sociais a partir da análise dos processos através dos quais os indivíduos, em interação social, constroem explicações sobre objetos sociais como, por exemplo, a saúde. Trata-se, portanto, do estudo científico do senso comum que, por sua vez, contribui para caracterização de uma identidade grupal, bem como a tomada de decisões relacionada aos comportamentos de risco e autocuidado ${ }^{(12)}$. Ante o exposto, pesquisas evidenciaram que, para a maioria dos adolescentes, saúde não tem o significado de "não adoecer", mas sim o quanto se pode fazer "vivendo coisas boas da vida" ${ }^{\prime(13,14)}$.

Para analisar o cenário dos fatores de risco de exposição ao VHB entre estudantes do ensino médio regular utiliza-se o modelo social de camadas ${ }^{(15)}$. É importante ressaltar que, no plano individual, a vulnerabilidade está relacionada a comportamentos que promovem a exposição aos riscos. No entanto, tais atitudes não devem ser entendidas apenas como uma ação voluntária das pessoas, mas também permeadas por condições objetivas do meio e aspectos culturais e sociais ${ }^{(16)}$.

O principal fator de risco à exposição ao VHB pelos estudantes do atual estudo correspondeu aos procedimentos de manicures/ pedicures realizados com materiais que podem estar contaminados com o vírus. A procura por serviços de embelezamento e higiene, nesta fase da vida, está relacionada à percepção da "beleza corporal" como valor ${ }^{(17)}$. Para não se tornarem excluídos, muitos vivem em função de melhorar o aspecto físico mesmo reconhecendo que a mídia constrói modelos de beleza difíceis de serem alcançados ${ }^{(18,19)}$. No aspecto intersetorial, é importante associar questões da biologia do vírus, como grande capacidade de sobrevivência, resistência e infectividade ${ }^{(1,20)}$, à baixa adesão das práticas de biossegurança nos estabelecimentos no que tange, principalmente, à esterilização dos materiais permanentes, como alicates e tesoura, e ao descarte dos materiais de consumo, que incluem lixas e palitos de madeira ${ }^{(21,22)}$. Nesse contexto, cabe ressaltar a existência de estudos que evidenciam a relação dos serviços de beleza e estética com a transmissão de hepatite $\mathrm{B}^{(23-25)}$.

Outro fator de risco para exposição ao VHB que apresentou uma frequência importante no presente trabalho foi a não utilização de preservativos durante as relações sexuais. No Brasil, a idade média do início da vida sexual vem se estabilizando desde 2000 e oscila, atualmente, entre 14 e 16 anos $^{(26)}$ - faixa etária que abrange uma grande parte da amostra. A epidemia da Síndrome da Imunodeficiência Adquirida e a elevação da incidência de gravidez na adolescência levou ao aumento do uso de preservativos entre jovens com 16 a 24 anos, tanto com parceiros eventuais quanto estáveis ${ }^{(26,27)}$.

A frequência de adolescentes que não utilizam regularmente preservativos variou muito de acordo com a região do país e método da pesquisa: 54,5\% em Bragança/São Paulo ${ }^{(28)}, 64 \%$ em Mato Grosso ${ }^{(29)}$ e 24,1\% em Teresina ${ }^{(30)}$. Dados da Pesquisa Nacional de Saúde Escolar mostraram que 75,9\% dos adolescentes usaram preservativos na última relação sexual ${ }^{(31)}$. Os fatores que mais influenciam no uso inconsistente do preservativo incluem confiança no parceiro a partir de um vínculo mais estável e maior envolvimento emocional, uso de outros métodos contraceptivos pelas meninas (o que pode refletir a atribuição da responsabilidade feminina exclusiva), preferência por parte do parceiro pela não utilização (o que evidencia a dificuldade de negociação sexual e a relação de poder entre gêneros), redução do prazer e do clima no momento do ato sexual ${ }^{(27,32-34)}$. Cabe ressaltar que a principal preocupação dos adolescentes é com a prevenção de gravidez, e não com DSTs ${ }^{(35)}$.

Em relação aos outros tipos de riscos, que foram bem reduzidos na amostra estudada, em torno de 3\% possui piercing e/ ou tatuagem e menos de $5 \%$ haviam recebido transfusão sanguínea/transplante ou tinham histórico de uso de drogas injetáveis. Observaram-se resultados similares em outras pesquisas ${ }^{(36,37)}$.

Um importante aspecto a ser trabalhado dos determinantes sociais de saúde, que abrange todos os fatores de risco, está relacionado à falta de conhecimento referente às formas de transmissão do VHB, especialmente por meio da exposição percutânea ou mucosas envolvendo sangue, pela maioria dos adolescentes ${ }^{(30,37)}$ e dos profissionais que atuam nos serviços de embelezamento e higiene ${ }^{(38,39)}$. Além disso, foi verificado que o principal nível de escolaridade dos pais era o ensino fundamental. Cabe ressaltar que os níveis de instrução, tanto materno quanto paterno, especialmente nos casos das crianças e adolescentes, podem influenciar o acesso à informação e o estímulo ao cuidado com a própria saúde ${ }^{(40)}$.

No caso da hepatite B, por ser uma doença sexualmente transmissível também, a educação sexual se torna um componente importante no contexto familiar. Um estudo realizado com 383 adolescentes solteiros identificou que tanto os indivíduos do 
sexo feminino quanto do masculino procuravam informações sobre sexo com amigos (aproximadamente $45 \%$ ), e outros (cerca de 14\%) não conversavam com ninguém. Em relação às dúvidas sobre doenças sexualmente transmissíveis, $35 \%$ dos estudantes procuravam profissionais de saúde e professores ${ }^{(41)}$.

Acredita-se que a população adolescente tem sido negligenciada, em sua grande parte, pelas campanhas de vacinação em massa, o que pode contribuir para a baixa cobertura vacinal e para o aumento da incidência de hepatites virais nessa faixa etária ${ }^{(7,42)}$. Tal fato é reforçado pela redução na procura por vacinas por adolescentes e jovens adultos quando o seguimento vacinal de rotina na Unidade Básica de Saúde se encerra, em geral, ao completar 5 anos de idade ${ }^{(43)}$.

Ao se considerar aspectos relacionados ao cartão de vacina e imunização contra o VHB, a maioria dos estudantes do presente estudo relatou que possuía o cartão de vacina e $42 \%$ afirmaram que eram vacinados. Ao se analisar o cartão de vacina de uma parcela dos estudantes da pesquisa (240), $88 \%$ estavam com esquema vacinal completo. Dessa forma, evidenciou-se que a situação vacinal identificada no presente estudo ainda é inferior aos $95 \%$ preconizados pelo Ministério da Saúde ${ }^{(30)}$. Pesquisas apontam uma alta resistência dos adolescentes susceptíveis a receber a primeira dose do imunógeno associada a diversos fatores. Em relação a não adesão, os principais motivos relatados foram falta de orientação, medo e desconsideração da importância da vacina. As justificativas para a presença do esquema vacinal incompleto incluíram esquecimento, falta de orientação sobre a necessidade de 3 doses e, até mesmo, o desconhecimento do motivo ${ }^{(44-46)}$.

Além disso, foi também observada uma redução dos cuidados e de conhecimento em relação à imunização, já que $23 \%$ relataram que não sabem ou não possuem o cartão de vacina e 58\% relataram desconhecer a situação vacinal ou não serem vacinados contra hepatite B. Esse resultado corrobora outro estudo, realizado no Piauí, no qual apenas 39,8\% dos adolescentes possuíam o cartão de vacina e a maioria desconhecia o calendário de vacina do adolescente ${ }^{(46)}$.

Nesse sentido, é importante discutir sobre as Oportunidades Perdidas de Vacinação (OPV), que, segundo a Organização Pan-Americana de Saúde, ocorre quando uma pessoa candidata à imunização, que não apresenta contra-indicações, comparece a um serviço de saúde e não recebe as vacinas necessárias ${ }^{(47)}$. A negligência dos profissionais de saúde em observar o cartão de vacinas dos usuários, entre outras atitudes negativas, tem se constituído em uma das principais causas de OPV, o que, por sua vez, se reflete na cobertura vacinal ${ }^{(40)}$.

Ressalta-se que um dos melhores momentos para atualizar o esquema vacinal contra hepatite B é entre 11 e 12 anos, pois nessa idade, além de ser mais fácil o controle pelos pais, o que facilita a adesão a esquemas de múltiplas doses, a maioria não iniciou a vida sexual ${ }^{(48)}$. Estudos destacam ainda que a escola é um local que facilita o cumprimento do esquema vacinal contra hepatite $\mathrm{B}^{(45)}$.

Diante do cenário acerca da imunização apresentado, é essencial o planejamento de estratégias de sensibilização dos adolescentes quanto ao cuidado com o cartão de vacina no momento da transição entre a fase da criança - na qual a mãe se encarrega do cuidado em relação ao cartão de vacina e atualização do mesmo - e a vida adulta - quando, muitas vezes, há exigências referentes à atualização vacinal para ingresso no mercado de trabalho e nas instituições de ensino superior. Evitando, assim, a aplicação de doses desnecessárias de algumas vacinas por falta do cartão ou por desconhecimento. Além disso, a sensibilização deve também ocorrer no sentido da importância da imunização contra a hepatite B, especialmente antes do início dos comportamentos de risco associados a essa faixa etária (atividade sexual desprotegida, uso de álcool e drogas injetáveis, maior preocupação com a autoimagem e procura por serviços de manicure, piercing e tatuagens).

No âmbito da educação em saúde, cabe ressaltar a importância de se considerar a percepção positiva dos adolescentes em relação à saúde para o planejamento das práticas educativas. No geral, essas práticas são fundamentadas apenas em noções de risco e conceitos negativos de saúde e podem, portanto, distanciarem-se do contexto real dos adolescentes e inferirem noções de autoridade e imposição. Pesquisadores destacam a frequência de abordagens reducionistas e normativas que são contrárias à perspectiva do autocuidado como um processo de percepção do mundo, das relações interpessoais, do agir de si para consigo, de modificar-se e transforma-se ${ }^{(49,50)}$. Considerando essas premissas, as atividades de promoção em saúde e prevenção de doenças devem ser vistas como mecanismo de intercâmbio entre os saberes científicos e populares por meio do estabelecimento de interações dialógicas horizontais e que estimulem a autonomia, atitudes críticas e escolhas responsáveis em relação ao cotidiano.

Uma possível limitação deste estudo está relacionada ao viés da dependência das declarações dos estudantes, uma vez que as respostas podem estar pautadas em situações idealizadas e não concretas. No entanto, observa-se uma distribuição de frequência relativa bem similar entre as diferentes regiões estudadas. A partir do reconhecimento dos principais fatores de risco e da situação vacinal dos estudantes do ensino médio, é importante o desenvolvimento de um estudo analítico considerando as características sociodemográficas e suas possíveis associações com os comportamentos de risco de exposição ao VHB.

Sugere-se ainda que o ambiente escolar seja um local que favoreça a identificação dos estudantes susceptíveis à hepatite B, atualização do cartão de vacina, bem como a implementação de estratégias de educação em saúde que despertem nos adolescentes a necessidade da imunização, tanto para sua vida atual quanto para a fase adulta. Portanto, torna-se essencial a promoção de práticas educativas para possibilitar a ampliação do conhecimento sobre a doença e minimizar os riscos de exposição ao vírus considerando as representações sociais dessa faixa etária e os determinantes sociais de saúde. 


\section{CONCLUSÃO}

A maioria dos estudantes do ensino médio regular da rede pública estudada está na adolescência e apresenta características sociodemográficas e comportamentos de risco que podem deixá-los mais expostos ao vírus da hepatite B. Procedimentos de manicures que não seguem as normas de biossegurança se destacaram no contexto da análise uma vez que mais da metade da amostra era do sexo feminino.

Observou-se também uma redução do cuidado em relação ao cartão de vacina, bem como um déficit de conhecimento da situação vacinal contra hepatite B. A taxa de imunização contra a doença se apresentou abaixo daquela preconizada pelo Ministério da Saúde.

\section{AGRADECIMENTOS}

Aos membros da Diretoria Regional de Educação 02 (DRE02) e aos estudantes que tornaram este estudo possível.

\section{REFERÊNCIAS}

1. Lopes TGSL, Schinoni MI. Aspectos gerais da hepatite B. R Ci Med Biol [Internet]. 2011 [acesso em 2015 Jun 6];10(3):33744. Disponível em: http://www.portalseer.ufba.br/index.php/cmbio/article/viewFile/5899/4251

2. Ministério da Saúde (BR). DATASUS [Internet]. Departamento de Informática do SUS [acesso em 2015 Jun 6]. Disponível em: http://www2.datasus.gov.br/DATASUS/index.php?area=02

3. Kiffer CRV, Viana GB, Cheinquer H. Hepatite B: epidemiologia. In: Focaccia R. Tratado de hepatites virais. São Paulo: Atheneu; 2002. p. 127-32.

4. Gogos CA, Fouka KP, Nikiforidis G, Avgeridis K, Sakellaropoulos G, Bassaris H, et al. Prevalence of hepatites B and C vírus infection in the general population and selected groups in South-Western Greece. Eur J Epidemiol. 2003;18(6):51-7.

5. Hahné S, Ramsay M, Baloqun K, Edmunds WJ, Mortimer P. Incidence and routes of transmission of hepatitis B vírus in England and Wales, 1995-2000: implication for immunisation policy. J Clin Virol. 2004;29(4):211-220.

6. Amaral VC. Hepatite B: risco ocupacional. Niterói: UFF; 2005.

7. Porto SO, Cardoso DD, Queiróz DA, Rosa H, Andrade AL, Zicker F, et al. Prevalence and risk factors for HBV infection among street youth in Central Brazil. J Adolesc Health. 1994;15(7):1-5.

8. Abuassi C. Imunização em adolescentes. Rev Hosp Univers Pedro Ernesto (UERJ). 2007; 6(1):34-41.

9. Ministério da Saúde (BR), Departamento de Vigilância das Doenças Transmissíveis. Nota informativa $n^{\circ}$ 149, de 2015/ CGPNI/DEVIT/SVS/MS [Internet]. Brasília; 2015 [acesso em 2015 Jun 6]. Disponível em: http://www.cvpvacinas.com. br/pdf/nota_informativa_149.pdf

10. World Health Organization. Problemas de la salud de la adolescência. Informe de un comité de expertos de la OMS [Internet]. Genebra: WHO; 1965 [acesso em 2015 Jun 6]. Disponível em: http://apps.who.int/iris/bitstream/10665/38485/1/ WHO_TRS_308_spa.pdf.

11. Santos FH, Andrade VM, Bueno OFA. Neuropsicologia hoje. $2^{\mathrm{a}}$ ed. Porto Alegre: Artmed; 2015.

12. Jodelet D. As representações sociais. Rio de Janeiro: UERJ; 2001.

13. Cromack LMF, Bursztyn I, Tura LFR. O olhar do adolescente sobre saúde: um estudo de representações sociais. Ciênc Saúde Coletiva. 2009;14(2):627-34.

14. Ferreira MA, Alvim NAT, Teixeira MLO, Veloso RC. Saberes de adolescentes: estilo de vida e cuidado à saúde. Texto \& Contexto Enferm. 2007; 16(2):217-24.

15. Dahlgren G, Whitehead M. Policies and strategies to promote social equity in health. Background document to WHO Strategy paper for Europe. Institute for Future Studies; 1991.

16. Meyer DEE, Mello DF, Valadão MM, Ayres JCRM. "Você aprende. A gente ensina?” Interrogando relações entre educação e saúde desde a perspectiva de vulnerabilidade. Cad Saúde Pública. 2006;22(6):1335-42.

17. Assis SG, Avanci JQ, Silva CMFP, Malaquias JV, Santos NC, Oliveira RVC. A representação social do ser adolescente: um passo decisivo na promoção da saúde. Ciênc Saúde Coletiva. 2003;8(3):669-80. 
18. Silva MLA, Taquette SR, Coutinho ESF. Sentidos da imagem corporal de adolescentes no ensino fundamental. Rev Saúde Pública. 2014;48(3):438-44.

19. Pavan C, Simonato P, Marini M, Mazzoleni F, Pavan L, Vindigni V. Psychopathologic aspects of body dysmorphic disorder: a literature review. Aesthetic Plast Surg. 2008;32(3):473-84.

20. Williams IT, Perz JF, Bell BP. Viral hepatitis transmission in ambulatory health care settings. Clin Infectious Dis. 2004; 38(11):1592-1598.

21. Melo FCA, Isolani AP. Hepatite B e C: do risco de contaminação por materiais de manicure/pedicure à prevenção. SaBios. 2011;6(2):72-8.

22. Oliveira FM, Alves AS, Santos LA, Santana TLS, Silva GM, Kameo SY. Adesão às medidas de biossegurança relacionada à hepatite B por manicures. Ensaios Ciências. 2014;18(2):83-90.

23. Johnson IL, Dwyer JJ, Rusen ID, Shanin R, Yaffe B. Survey of infection control: procedures at manicure and pedicure establishments in North York. Can J Public Health. 2001;92(2):134-7.

24. Hepworth J, Murtagh M. Correct procedures and cutting corners: a qualitative study of women's occupational health and safety in a beauty therapy industry. Aust New Zealand J Public Health. 2005;29(6):555-7.

25. Mariano A, Mele A, Tosti ME, Parlato A, Gallo G, Ragni P, et al. Role of beauty treatment in the spread of parenterally transmitted hepatitis viruses in Italy. J Med Virol. 2004;74(2):216-20.

26. Paiva V, Calazans G, Venturi G, Dias R. Idade e uso de preservativos na iniciação sexual de adolescentes brasileiros. Rev Saúde Pública. 2008;42(Supl 1):45-53.

27. Almeida MC, Aquino EM, Gaffikin L, Magnani RJ. Contraceptive use among adolescents at public schools in Brazil. Rev Saúde Pública. 2003;37(5):566-75.

28. Cruzeiro ALS, Souza LDM, Silva RA, Pinheiro RT, Rocha CLA, Horta BL. Comportamento sexual de risco: fatores associados ao número de parceiros sexuais e ao uso de preservativo em adolescentes. Ciênc Saúde Coletiva. 2010;15(Supl 1):1149-58.

29. Duarte SJH, Urel DR, Zorman IBS, Alexandre MG, Ravagnani CFC. A prática de autocuidado à saúde na perspectiva dos adolescentes. Rev Enferm UFPE. 2014;8(5):1290-9.

30. Araújo TME, Carvalho AMC, Monteiro RM. Análise da vulnerabilidade dos adolescentes à hepatite B em Teresina/PI. Rev Eletrônica Enferm. 2012;14(4):873-82.

31. Malta DC, Silva MAI, Mello FCM, Monteiro RA, Porto DL, Sardinha LMV, et al. Saúde sexual dos adolescentes segundo a Pesquisa Nacional de Saúde dos Escolares. Rev Bras Epidemiol. 2011;14(1):147-56.

32. Alves AS, Lopes MHBM. Conhecimento, atitude e prática do uso de pílula e preservativo entre adolescentes universitários. Rev Bras Enferm. 2008;61(1):11-7.

33. Visser R, Smith A. Relationship between sexual partners influences rates and correlates of condom use. AIDS Educ. 2001;13(5):413-27.

34. Vieira MAS, Guimarães BEM, Barbosa MA, Turchi MD, Alves MFC, Seixas MSC, et al. Fatores associados ao uso do preservativo em adolescentes do Gênero feminino no município de Goiânia. J Bras Doenças Sex Transm. 2004;16(3):7783.

35. Borges ALV, Schor N. Início da vida sexual na adolescência e relações de gênero: um estudo transversal em São Paulo, Brasil, 2002. Cad Saúde Pública. 2005;21(2):499-507.

36. Schmidt M, Middleman AB. The importance of hepatitis B vaccination among adolescents. J Adolesc Health. 2001;29(3):217-22.

37. Livramento A, Cordova CMM, Spada C, Treitinger A. Avaliação do nível de conhecimento de adolescentes a respeito da transmissão e prevenção das hepatites C. Rev Patol Trop. 2009;38(3): 155-63.

38. Oliveira ACDS, Focaccia R. Survey of hepatitis B and C infection control: procedures at manicure and pedicure facilities in São Paulo, Brazil. Braz J Infect Dis. 2010;14(5):502-7.

39. Hellard M, Aitken C, Mackintosh A, Ridge, Bowden S. Investigation of infection control practices and knowledge of hepatitis C among body-piercing practitioners. Am J Infection Control. 2003;31(4):215-20. 
40. Araújo TME, Sá LC, Silva AAS, Costa JP. Cobertura vacinal e fatores relacionados à vacinação dos adolescentes residentes na área norte de Teresina/PI. Rev Eletrônica Enferm [Internet]. 2010;12(3):502-10.

41. Borges ALV, Nichiata LYI, Schor N. Conversando sobre sexo: a rede sociofamiliar como base de promoção da saúde sexual e reprodutiva de adolescentes. Rev Latinoam Enferm. 2006;14(3):422-7.

42. Melo MCP, Santos MM, Mendes RNC, Sales JRP, Silva RM. Percepção de adolescentes sobre imunização em uma escola pública de Petrolina - PE. REME Rev Min Enferm. 2013;17(2):374-80.

43. Rodrigues IC, Fioravante IO, Kubota RMM, Furtil AP, Justino STS, Santos MR. Vacinação de escolares: estimulando o autocuidado e a responsabilização. Arq Ciênc Saúde. 2011;18(4):170-5.

44. Francisco PMSB, Donalisio MR, Gabriel FJO, Barros MBA. Vacinação contra hepatite B em adolescentes residentes em Campinas, São Paulo, Brasil. Rev Bras Epidemiol. 2015;18(3):552-67.

45. Oliveira MDS, Paggoto V, Matos MAM, Kozlowski AG, Silva NR, Junqueira ALN, et al. Análise de fatores associados à não aceitação da vacina contra hepatite B em adolescentes escolares de baixa renda. Ciênc Saúde Coletiva. 2007;12(5):124752.

46. Carvalho AMC, Araújo TME. Fatores associados à cobertura vacinal em adolescentes. Acta Paul Enferm. 2010; 23(6):796802.

47. Organizacións Panamericana de la Salud. Oportunidades perdidas de vacunación em las Américas: diagnóstico y intervenciones, 1988-1990 [Boletim informativo]. PAI. 1991;13(3).

48. Coutinho MFG. Adolescência: vacina contra hepatite B. Adolec Saúde. 2010;7(1):23-30.

49. Gomes CM, Horta NC. Promoção da saúde do adolescente em âmbito escolar. Rev APS. 2010;13(4):486-99.

50. Bub MBC, Medrano C, Silva CD, Wink S, Per-Erik L, Santos EKA. A noção de cuidado de si mesmo e o conceito de autocuidado na enfermagem. Texto \& Contexto Enferm. 2006;15(Esp):152-7.

\section{Endereço primeiro autor:}

José Marcos de Jesus Santos

Universidade Federal de Sergipe - UFS

Campus Prof. Antonio Garcia Filho - Departamento de Enfermagem.

Av. Governador Marcelo Dedá, 13

Bairro: Centro

CEP: 49400-000 - Lagarto - SE - Brasil

\section{Endereço para correspondência:}

Flávia Márcia Oliveira

Av. Governador Marcelo Déda, s/n.

Bairro: São José

CEP 49400-000 - Lagarto - SE - Brasil

E-mail: fmo.ufs@hotmail.com 